\title{
Research on the Current Situation and Strategy of Talent Training of Logistics Management Specialty from the Perspective of Intelligent Logistics
}

\author{
Xinhong Deng ${ }^{1, a}$ \\ 'Jiangxi Vocational Technical College of Industry \& Trade, Nanchang, Jiangxi,330038, China \\ a739960103@qq.com
}

\begin{abstract}
In the current development of information internet life, intelligent logistics is developing rapidly in the field of logistics industry. As an emerging development direction in the field of logistics, the development of intelligent logistics often needs more excellent logistics management professionals. This paper mainly explores the cultivation of logistics management talents in Colleges and universities from the perspective of intelligent logistics. From the current development of smart logistics, its own foundation is very rigorous, so it must continue to develop in combination with excellent logistics management professionals. Therefore, talent training must be regarded as the primary task of reform, so that the logistics industry can be transformed in the era of Internet information.
\end{abstract}

Keywords: intelligent logistics, physical distribution management, personnel training

\section{智慧物流视角下物流管理专业人才培养现状与策略探 究 \\ 邓新红 ${ }^{1, a}$}

\section{${ }^{1}$ 江西工业贸易职业技术学院 江西 南昌 330038 \\ a739960103@qq.com}

摘要:

在当下发展的信息互联网生活中，智能物流在物流产业的领域中发展迅速，作为物流领域的新兴发展方向， 智能物流的发展往往需要更加优秀的物流管理专业人才。本文主要探究在智能物流的视角下，高校对于物流 管理人才的培养。从目前的智慧物流发展状况来看，其本身的基础是十分严谨的，所以必须结合优秀的物流 管理专业人才继续发展, 因此就必须把人才培养作为改革的首要任务, 才能够让物流产业在互联网信息时代 下进行转型。

关键词: 智慧物流; 物流管理; 人才培养

\section{1. 智慧物流的内涵与特点}

当下，我国物流业已迈人新的发展阶段, 越来越 多的企业也开始拥抱技术革新。但企业要发展, 人 才是先导。人才作为企业核心竞争力的第一要素, 物流企业在新发展动态下对人才的需求也在发生巨 大变化。因此, 在深刻理解智慧物流内涵与特点的 基础上, 探索满足智慧物流需求、对接区域经济发
展的物流专业人才培养问题, 解决智慧物流发展中 的人才智库问题, 对加快推进智慧物流发展至关重 要。

物流产业的发展来说，智慧物流几乎成为了当 代的发展前景，所以智慧物流正是在以互联网基础 的前提下，将物流领域中网大数据与云计算的技术 结合起来深度运用到物流产业的发展当中，使得物 流产业能够进行智能化，成为整体上提高物流产业 的运作效率。 


\section{1. 智慧物流的内涵}

从物流产业进行发展以来,一直都是实体经济 的重要组成部分之一, 因此想要真正意义上的把物 流产业进行高质量的发展, 那么就必须从物流产业 本身的运作进行转型升级。随着市场的需求逐渐扩 大, 人们对于物流产业的运输效率也逐渐进行一定 的速度要求, 因此将物流产业中发展智能物流是能 够有效的推进物流产业的整体效益发展, 并且物流 产业的发展能够有效的加强我国世界经济的活力, 从而可以在一定程度上响应国家深化供给侧改革的 政策。并且物流产业的智能化不仅仅能够在效率性 能提高的同时, 也能够有效的把物流产业的质量做 好，对于高质量的物流产业的发展来说，将信息化， 标准化, 数字化系统, 化智能化作为整体特征的智 慧物流, 正好能够最大程度上的满足当代市场的需 求以及人们的需求。

\section{2. 特点}

随着科技的进步技术的发展物流企业，从很大 程度上慢慢开始依赖互联网技术, 在互联网信息时 代中, 互联网技术往往包括物联网, 云计算人工智 能等新兴技术, 将这些新技术有效运用到物流产业 的每一个环节当中, 比如在物流产业的操作环节当 中, 我们可以设置传感器, 增加摄像头, 通过 GPS 装备进行定位等等, 都能够很大程度上把物流产业 运行过程中所产生的实时数据进行收集, 从而通过 云计算能力进行分析, 把每一个物流节点最优质的 运输通道决策出来。

通过这样的技术, 能够把整个物流系统进行资 源的有效利用和集成化运作, 从而把物流管理的标 准整体上提高, 这其中最大的优点是能够把最恰当 的货物在最优质的路径和成本最低的方法而送到合 适的目的地, 不仅仅在很大程度上降低了成本, 还 可以在最快的时间内把物资送到。所以这个物流可 以从很大程度上推动整个物流产业的发展, 这种发 展往往是质变的, 这样的转型不仅仅有利于物流领 域的创新, 才能够很大意义上的增加国家的实体经 济能力。

\section{2. 智慧物流视角下物流管理专业人才培养现 状}

智慧物流视角下物流管理专业人才的培养现状 下暴露了很多问题, 其中比较突出的问题是课程体 系设置与智慧物流人才素质需求不匹配; 以及教学 手段与实训条件和师资力量不能满足智慧物流人才 培养需求等问题。

\section{1. 课程体系设置与智慧物流人才素质需求 不匹配}

面对当前高校课程存在的物流管理课程来说， 难以培养出当下智能物流发展企业想要需要的综合 性人才, 这种多学科知识以及多专业技能掌握的高 素质复合型人才, 往往是大部分高校的专业课难以 进行培养的, 因为在高校的专业知识学习过程中, 对于人才的专业培养定位是十分确定的, 这种不确 定性往往取决于课程体系的制定随意以及不严谨的 诸多问题存在。

当前高校的课程体制设置上最主要的问题是难 以培养出智慧物流人才组织所需要的标准, 专业发 展的主要方向不准确, 以及课程体系当中很多就业 岗位的正确课程内容, 难以和学生自身想要培养的 职业能力不匹配, 都是导致高校无法培育出当代智 能物流所需要人才的重要原因。

\section{2. 教学手段与实训条件和师资力量不能满 足智慧物流人才培养需求}

首先当前高校的教学手段, 由于执行条件往往 都难以满足, 对于物流人才的培养需求, 在这一点 上高校的实训条件几乎没有真正意义上的让高校物 流实训得到完整的训练, 对症情况的出现, 就会导 致学生在进行物流管理学习的过程中实践经验不足, 没有对物流管理行业中所需要用到的各种系统进行 使用, 白高山目前存在的软件培训是难以和真实的 事情拉近距离的。其次毕业生在高校进行专业知识 与技能学习过过程中, 难以学到智能物流中所需要 的各种知识与能力, 导致毕业生在未来出去寻找工 作的同时难以满足当下智能物流企业的各种需求, 从而产生就业压力。

最后主要在于学校本身的师资力量很多, 专业 课老师没有在进行智能物流技术的同时，本身就没 有具体的实战经验, 所以难以对课程进行相应的改 造以及建设, 学校的教师群体也难以根据当前的物 流发展状况来进行课程的调整, 从而让学生错过了, 对于新兴知识学习的机会, 这种情况的出现, 几乎 让高校难以培养出物流企业高质量发展, 在物流产 业环境的发展过程中, 除了本专业的各种专业知识 理论, 还需要懂得更多领域的扩展性技能与知识, 才能够让学生更好的适应未来智能物流企业的各种 技能需求, 因此高校在这方面的实施力量就十分欠 缺, 难以从教师引导方面对学生进行培训。

\section{3. 智慧物流视角下物流管理人才培养策略}

为了更好的在智慧物流视角下培养好高素质的 物流管理人才, 需要有关部分组织机构从这一下路 径考虑。 
$\mathrm{zZ}$ 分别是想办法以关键能力为核心，提升人才 综合素养; 以产业需求为导向，明确人才培养目标; 以信息化为手段，提高人才培养质量; 以教师队伍 建设为抓手, 为人才培养提供智力支持; 以及做好 以校企合作为途径, 完善实践教学条件。

\section{1. 以关键能力为核心 提升人才综合素养}

对于现存的智慧物流来说, 想要对其进行发展 和建设就必须需要将关键能力掌握的人才进行培养。 当前的互联网信息技术，任何的事物都是以数据化， 因此掌握关键能力的高质量综合性人才, 往往都是 以技术和数据作为本身技能发展基础的。当前企业 所大量需求的高质量复合型人才都需要学校在人才 培养上教学生的专业知识与实践积累都结合起来.

不仅如此, 对于掌握关键技能核心的高素质人 才, 还需要具有良好的职业道德以及健康的心理。 高校在培养高质量中国人才的同时, 必须注重以学 生为主体的培养核心, 将关键能力作为培养方案的 重要手段, 让学生在能够把关键能力学会同时也能 够把物流产业本身的专业的课程进行优质学习, 从 而让学生在面临专业性知识的问题上有着自我解决 的能力, 并且在与人沟通方面和思维创新方面。都 需要把这些方面的各个关键能力都进行培养, 才能 够真正意义上培养出物流企业所需要的高质量综合 型应用人才, 而且对于这样的高素质人才, 往往可 以有效的把物流产业进行创新和推动, 成为在一定 程度上是有生发展力量中的可持续发展能力。

\section{2 . 以产业需求为导向 明确人才培养目标}

对于任何企业来说, 市场的需求往往就等于产 业的需求, 因此要想企业能够有效的进行实体经济 的发展, 就必须把产业需求作为导向, 在对人才进 行招聘的过程中, 要求具体的人才, 必须有扎实的 基础知识以及优质的实践经验积累, 才能够把人才 投入到产业需求的发展中, 满足产业的具体需求, 促进产业的整体经济效益发展。高校在这个过程中 需要做到的是, 让学生能够有这个明白产业需求的 方向, 比如跨境电商总裁的电商以及各大电商领域 的发展都是在高校了解市场需求和企业进行参考后 对人才进行培养的重要因素。

信息化时代的世界消化数据往往都是实质数据, 将这些数据进行云计算和大数据分析, 我能够有效 的吧, 智能物流进行可持续性发展, 将采用的需求 满足是需要各大人才进行培育和创新, 才能够对其 漏洞进行评估, 满足需求量。高校在与企业进行合 作的过程中, 能够将好像顾城的物流专业人才培养 计划经济调整, 让高校能够有效的培育出企业需要 的复合型专业应用人才。

同时, 在课程体系设置上, 要对接职业岗位要 求, 组建多方教学专家团队, 重构专业课程体系,
整合和精简原有课程, 增设适应智慧物流需要的物 联网技术、通信技术、云计算、人文教育和创新创 业教育等课程, 增大实践性教学课时占比, 重视知 识应用和实践能力培养。

\section{3. 以信息化为手段 提高人才培养质量}

以信息化带动教育现代化越来越受到学校的高 度重视, 但信息化技术在教学中应用, 不仅仅是对

“黑板 + 粉笔” 的简单代替。教育信息化带来的是 教育形式和学习方式的重大变革。从深层次说, 它 倡导的是一种自主式、探究式、网络化的泛在学习 方式, 打破学习的时空限制, 变被动式学习为主动 式学习, 让学生成为学习的主人。借助开放的信息 化教学平台, 学生可以根据个人兴趣爱好, 便捷地 获取所需知识, 开展自主学习, 扩充知识面, 夯实 专业基础, 明确发展方向, 主动适应人才需求的新 变化。其次, 借助信息化平台, 还可以实施学分互 认制度或开展课程外包。对于物流管理专业资源不 足的高校来说, 通过利用同行业优秀的教育资源和 高质量的实训条件, 可以扩充师资力量, 改善教学 质量。此外，系院之间、高校之间优质资源流动融 合共享, 也有利于相互协作共同开发出更优质高效 的课程资源，从而提高整体的人才培养质量。

\section{4. 以教师队伍建设为抓手 为人才培养提供 智力支持}

针对目前高校培育高质量专业综合人才的方式 来看, 最主要的培训力量还是来自于教师队伍的建 设。教师群体也是高校发展的重要基础, 因此高校 想要培育出高质量的综合性人才, 就必须引入和培 育出高水平的教师队伍, 综合真正意义上的满足智 能物流, 高质量复合型, 有人才的培养目标。

因此对于现存的学校是指队伍建设主要可以从 以下几个方向改变: 一是高校必须重视加强教师本 身的能力建设与培养, 在现存的他的高校中学生难 以通过自身的学习与发展, 把课堂中的专业知识与 技能进行学习, 因此高校教师水平必须进行自我学 习, 把本身的知识储备提高, 将目前的课程进行一 定的改革, 有效运行经济, 把教师团队的科研能力 提升，用资金或者各种荣誉鼓励教师走出课堂，在 物流管理专业中得到有效的实践经验积累。

但是高校可以通过引进先进人才的拜拜，把优 秀的人才引入专业的教师队伍中进行本校专业老师 的各个技能培训, 从而让教师队伍的整体实力上升, 把每一个学生群体的学习能力都顾及到位, 从而再 提升学生整体的综合素质, 同时也能够有效的把教 师队伍整体水平提高。三是根据需要聘请企业高水 平的技术人才和管理人才担任客座教授或教学实践 指导教师, 弥补应 用型课程的师资短缺, 增强实践 教学能力, 为智慧物流人才培养提供人才保障和智 力支持。 


\section{5. 以校企合作为途径 完善实践教学条件}

校企合作一直都是高校能够有效培养高质量人 才的重要途径, 对于高校的人才培育计划而言, 能 够与高校进行合作, 往往能够在了解高校所需求的 条件过程中，对人才进行培育计划的共同挑战，而 且对于高校应用型人才的培育来说，我也不太专业, 知识的学习以及实践教学的训练, 因此有效地完善 实践教学条件, 能够针对性地完成企业的人才需求, 从而推动学校整体专业的教育事业发展, 但是在这 个过程中往往需要大量资金的投入来进行基础设施 建, 因此高校教育事业发展过程中, 对于与企业的 发展合作是十分重要的。

校企合作不仅能够通过各个方式来对学生来进 行培养, 如高校能够在对课程进行改革的同时, 让 学生能够更好的学到知识与能力, 同时在实践中进 行知识的反馈, 双向人才的发展也出现, 也正是消 极合作所带来的现象。

\section{4. 结论:}

总之, 对于当前物流管理人才的培育是发展智 慧物流的主要方向, 优秀人才的培养有利于把智慧 物流进行更新与发展, 将智慧物流本身作为未来人 才的发展方向可以有效推动物流领域往数字化、智 能化方向发展, 这对于当下的物流企业来说提升企 业人才队伍的综合素质能够更有效占领市场经济高 地, 从而整体上使得现存的物流业运作做到迅速且 准确, 同时智慧物流的人才培育也为物流专业的大 学生提供了良好的工作岗位。

\section{REFERENCES}

[1] Jiang Qingmiao, Liu Haiqing, Zhuang Lingfeng, Chen Qinghua, hang Guilan, Qian Qingrong. Research on the recycling of express plastic packaging materials in China under green logistics [J]. Renewable resources and circular economy, 2020,13 (12): 14-18

[2] Qiu Zhipeng, Xu Zehao, Su haoxuan, Liang Zihao. Research on express packaging recycling model based on "green logistics" -- Taking Colleges and universities in Guangzhou as an example [J]. National circulation economy, 2020 (32): 9-11

[3] Zhang Jingya, Hou Ruhui, manor, Wang yingyue, Xie Yangyang, Wei zhuanling. Innovation and Research on express packaging recycling system from the perspective of logistics [J]. Mall modernization, 2020 (11): $18-20$

[4] Ying Qi, Zhang Chen, Wu Jinpeng, Huang Yanan, Zhao Zhenzhen, he Shi. Discussion on human factors restricting the development of express packaging recycling system and countermeasures [J]. Green packaging, 2020 (8): 57-60

[5] Wu Xiaodan, Liu can, Tang Jiajia, Li Ting, Zhou Rui. Research on mode innovation and optimization strategy of express packaging under the background of green logistics $[\mathrm{J}]$. Logistics engineering and management, 2020,42 (7): 14-17

[6] Liu Xinmeng, Wei Zirui, Wang LV Manlin. Research on intelligent packaging of Internet of things with the goal of green logistics [J]. Computer knowledge and technology: Academic Edition, 2019,15 (12): 251-252

[7] Yu Yao, Lin Lusuo. Legal regulation on the recycling of plastic packaging products in contact with food in China: Taking PET beverage bottles as an example [J]. Food science, 2019,40 (19): 370-377

[8] Gong Miaomiao. Research on the development path of China's green packaging industry -- from the perspective of coordinated development of circular economy and low-carbon economy [J]. Renewable resources and circular economy, 2018,11 (4): 9-12

[9] Zhang Wenmiao, Chen man, Geng Wenlong, Yang Mei, Zeng Yitang, Lu Hongxia. Research on recycling and reuse of express packaging based on the concept of sustainable development [J]. Ecommerce, 2017,0 (4): 36-37

[10] Zhang Tao, Tu Chao. Game Analysis of enterprises participating in Green Logistics Industrial Park Based on the intervention of environmental protection organizations $[\mathrm{J}]$. Logistics engineering and management, 2017,39 (12): 118-120

[11] Xu Yi, Meng Liqing, Wang Hao, Feng Jingiing. Research on integrated reverse logistics of waste wood materials based on o2o mode [J]. Logistics technology, 2017,40 (9): 50-52

[12] Zhu Lei, Li mengye, Du Yanping, Zhang Yuan, Wang Zhaohua, Li Ping. Research on circular packaging sharing system and its recycling mode in express industry [J]. Logistics technology, 2017,36 (9): $21-26$

[13] Cai Shaohong, Zhu MINLING, Wei Wenyun. Organization mode and operation mechanism of green logistics industry -- from the perspective of virtual industry cluster $[\mathrm{J}]$. Business economic research, 2017,0 (6): 84-85

[14] Gong Miaomiao. Research on the development path of China's green packaging industry -- from the perspective of coordinated development of circular economy and low-carbon economy [J]. Renewable resources and circular economy, 2018,11 (4): 9-12 
[15] Kong Jili, Ma Likun, Feng Ailan. Research on enterprise logistics management curriculum system for innovative application personnel training based on happy teaching concept $[\mathrm{J}]$. Logistics engineering and management, 2014 (1): 233-235

[16] Zhai Rongbing. Research on the reform of logistics management specialty in Local Applicationoriented Universities -- Thinking under the background of "excellent engineer education and training plan" [J]. Management and technology of small and medium-sized enterprises, 2013 (34): 217-218 